\title{
THE PHYSICAL ORIGINS OF THE MORPHOLOGY-DENSITY RELATION: EVIDENCE FOR GAS STRIPPING FROM THE SLOAN DIGITAL SKY SURVEY
}

\author{
ArJen van der Wel ${ }^{1}$, Eric F. Bell ${ }^{1,2}$, Bradford P. Holden ${ }^{3}$, Ramin A. SkibBa ${ }^{1,4}$, And Hans-Walter Rix \\ ${ }^{1}$ Max-Planck Institute for Astronomy, Königstuhl 17, D-69117 Heidelberg, Germany \\ 2 Department of Astronomy, University of Michigan, 500 Church Street, Ann Arbor, MI 48109, USA \\ ${ }^{3}$ University of California Observatories/Lick Observatory, University of California, Santa Cruz, CA 95064, USA \\ ${ }^{4}$ Steward Observatory, University of Arizona, 933 North Cherry Avenue, Tucson, AZ 85721, USA \\ Received 2009 November 6; accepted 2010 April 2; published 2010 April 22
}

\begin{abstract}
We provide a physical interpretation and explanation of the morphology-density relation for galaxies, drawing on stellar masses, star formation rates, axis ratios, and group halo masses from the Sloan Digital Sky Survey. We first re-cast the classical morphology-density relation in more quantitative terms, using low star formation rate (quiescence) as a proxy for early-type morphology and dark matter halo mass from a group catalog as a proxy for environmental density: for galaxies of a given stellar mass the quiescent fraction is found to increase with increasing dark matter halo mass. Our novel result is that-at a given stellar mass-quiescent galaxies are significantly flatter in dense environments, implying a higher fraction of disk galaxies. Supposing that the denser environments differ simply by a higher incidence of quiescent disk galaxies that are structurally similar to star-forming disk galaxies of similar mass, explains simultaneously and quantitatively these quiescence-environment and shape-environment relations. Our findings add considerable weight to the slow removal of gas as the main physical driver of the morphology-density relation, at the expense of other explanations.
\end{abstract}

Key words: galaxies: clusters: general - galaxies: elliptical and lenticular, $\mathrm{cD}$ - galaxies: formation - galaxies: fundamental parameters - galaxies: general - galaxies: statistics - galaxies: structure

Online-only material: color figures

\section{INTRODUCTION}

It has been known for many decades that galaxy morphology and environment are correlated (Smith 1935; Zwicky 1942; Sandage 1961). In dense environments the fractions of elliptical and S0 galaxies are higher than in low-density environments, at the expense of a decreased fraction of spiral and irregular galaxies (Dressler 1980; Vogt et al. 2004). This trend is universal and persists over a large dynamic range in density (Postman \& Geller 1984). S0 and spiral galaxies have in common that they have rotationally supported stellar disks. This suggests that there may be a direct evolutionary link, in the sense that S0 galaxies could be the descendants of spiral galaxies that had their star formation activity truncated.

The existence of the morphology-density relation (MDR) suggests that this process may be related to the environment (see, e.g., Boselli \& Gavazzi 2006, for an overview of the field). Such a process generally involves the partial or entire removal of the gaseous interstellar medium from galaxies that become satellites in larger dark matter halos, through interaction with the intergalactic medium. Fast, rather violent stripping of a galaxies' entire interstellar medium is often considered a viable option (Gunn \& Gott 1972; Quilis et al. 2000), but also more gentle stripping of hot gas outside the cold disk (Larson et al. 1980; Bekki et al. 2002; McCarthy et al. 2008), often called "starvation" or "strangulation," and the gradual stripping of neutral gas from the outer parts of disks (e.g., Chung et al. 2007) are commonly invoked. In any case, gas-deficient spiral galaxies in clusters do exist (e.g., Giovanelli \& Haynes 1985), which indicates that gas stripping occurs. Eventually, in all these scenarios, gas stripping removes the fuel for star formation, presumably producing a quiescent galaxy which is similar to a normal spiral galaxy in terms of its structural properties such as its bulge-to-disk ratio. Early on, van den Bergh (1976) described such gas-free, "amenic" spirals, and Balogh et al. (1998) observed how cluster galaxies have lower star formation rates than field galaxies with the same bulge-to-disk ratio.

However, if S0s occur when a spiral galaxy is stripped from its gas by interaction with the intragalactic medium, how do we explain the ubiquitous presence of S0s outside dense environments? Moreover, over the years, it has become clear that So galaxies differ from the spiral galaxies in several important ways, implying that the S0 population as a whole is not simply a population of disk galaxies with little or no star formation. S0 galaxies have more pronounced thick disks than spiral galaxies, as was first shown by Burstein (1979). Furthermore, the bar fraction among S0s is significantly smaller than that among spiral galaxies (Aguerri et al. 2009; Laurikainen et al. 2009). Perhaps the most important difference is that S0s have larger bulge-to-disk ratios than spiral galaxies (Dressler 1980). This is supported by increasingly convincing evidence that the Tully \& Fisher (1977) relation for S0 galaxies differs from that of spiral galaxies (Neistein et al. 1999; Hinz et al. 2003; Bedregal et al. 2006; M. J. Williams et al. 2010, in preparation). Although some of these differences may be the result of secular evolution and evolving stellar populations after the truncation of star formation, difficulties remain (see, e.g., Dressler 1984, for a discussion). In particular, the large bulges of S0 galaxies cannot be explained.

Besides the disconnect between S0s and spirals, the increased fraction of elliptical galaxies in dense environments (Dressler 1980) also challenges the idea that gas stripping explains the MDR. This concern, as well as the different properties of spirals and SOs, could be accommodated by a invoking a process that affects both the structure and gas content of a galaxy. Tidal interactions with other galaxies or the potential of a large halo can strip an infalling galaxy from gas and reduce the stellar disk as well (Moore et al. 1996). 
There are two reasons why it is surprisingly difficult to interpret the MDR, constrain its origin, and understand its implications for galaxy formation and evolution in general. First, "morphology" is a phenomenological parameter that is the combination of several physical quantities (structure, that is, bulgeto-disk ratio or concentration, and star formation activity). Second, many galaxy properties depend on one another, and some of these dependencies are much stronger than the dependency of morphology on environment. More specifically, although structure and star formation activity depend strongly on one another (e.g., Kauffmann et al. 2003, 2006; Hogg et al. 2004), these two parameters behave distinctly different as a function of environment. Kauffmann et al. (2004) and Blanton et al. (2005) showed that structure depends only weakly on environment, whereas star formation activity, usually as traced by color, decreases significantly from low- to high-density environments (e.g., Lewis et al. 2002; Hogg et al. 2003, 2004; Gómez et al. 2003; Balogh et al. 2004; Blanton et al. 2005; Baldry et al. 2006; Skibba \& Sheth 2009). Moreover, Balogh et al. (1998) showed that galaxies that are similar in structure have lower star formation rates if they are situated in a cluster. The net effect of these correlations is that the morphological mix changes with environment (van der Wel 2008; Bamford et al. 2009; Skibba et al. 2009).

To summarize the above, we have an apparent contradiction: on the one hand, the structural properties of galaxies do not show strong environmental dependencies; on the other hand, the relative elliptical, S0 and spiral galaxy fractions clearly do vary with environmental density, and these different types of galaxies have different structural properties. In this paper, we address this issue. We explicitly address the question whether gas stripping can explain the MDR, even in the face of the evidence that S0s and spiral galaxies are structurally different, that S0s occur in both low- and high-density environments, and that elliptical galaxies also prefer dense environments. Perhaps contrary to expectation, the listed evidence does not rule out gas stripping as an explanation for the MDR. One should distinguish between the S0 population as a whole, and those galaxies that have been affected by environmental processes, giving rise to the MDR. In other words, the origin of S0 galaxies and the origin of the MDR are not necessarily the same. This distinction, which was already noted by Postman \& Geller (1984), will prove to be critical.

In this paper, we examine the shapes of a large sample of galaxies with low star formation activity (quiescent galaxies), as inferred from their spectroscopic properties, in different environments. The sample selection from the Sloan Digital Sky Survey (SDSS) is described in Section 2. The shape parameter we use is the projected axis ratio. For an individual galaxy this may not contain much information, but for large samples it becomes a powerful diagnostic. We take advantage of the uniform and well-calibrated data set provided by the SDSS, which is ideally suited to disentangle projection effects and the intrinsic shape distribution of galaxies, as has previously been demonstrated by Vincent \& Ryden (2005) and Padilla \& Strauss (2008). Such an approach is complementary to studies of morphology, defined either visually or otherwise, because it makes no assumptions about the connection with galaxy structure. Because, in addition, we select our sample spectroscopically, our analysis is completely independent of the structural properties of different types of galaxies.

The first goal of this paper is to confirm that the decreased star formation rate in dense environments is reflected as an increased fraction of quiescent galaxies in massive dark matter halos (Section 3.1). A halo-based description of environment is more physical than estimates of the local surface number density of galaxies, and, moreover, the correlation between galaxy color and halo mass, and not galaxy density, has been demonstrated to be the principal driving factor behind the observed correlation between galaxy color and environment in general (Blanton \& Berlind 2007).

The second goal is to determine whether or not gas stripping can account for the dependence of star formation activity on group mass. We analyze the axis ratio distribution of quiescent galaxies and its dependence on halo mass, in Sections 3.2 and 3.3. Gas stripping of spiral galaxies, which presumably leaves their structural properties mostly unchanged, and alternative scenarios invoked to explain the MDR, such as harassment, which does change galaxy structure, will have a different effects on the axis ratio distribution of quiescent galaxies in high-mass halos.

Finally, we discuss our findings in the context of previous work and identify slow gas stripping as the process that is likely responsible for shaping the MDR (Section 4). This claim is then explicitly shown to be consistent with the different properties of spiral and S0 galaxies, and the increased fraction of elliptical galaxies in dense environments.

\section{SAMPLE}

We select a sample of quiescent galaxies from Data Release 6 of the SDSS (Adelman-McCarthy et al. 2008). Our sample includes galaxies at redshifts $0.04<z<0.08$ without detectable $[\mathrm{O}$ II] and $\mathrm{H} \alpha$ emission lines. The selection criteria are described and motivated in full by Graves et al. (2009b); but as opposed to that work, we do not exclude galaxies with a low concentration index and galaxies that are fit better by an exponential profile than by a de Vaucouleurs (1948) profile, because this may exclude quiescent, yet disk-like galaxies. As a consequence, our sample may include galaxies with star formation in an extended disk outside the SDSS spectroscopic fiber which has a 3 arcsec diameter. This, however, does not compromise our analysis as the typical galaxy has a size that is similar to the spectroscopic aperture. Less than $20 \%$ of the galaxies in our sample have sizes that are more than twice this aperture. The exclusion of all galaxies with emission lines also excludes quiescent galaxies with active nuclei. Their number, however, is small, and make up a small fraction of the population (e.g., Pasquali et al. 2009) that is negligible for our purposes.

The axis ratios were obtained as described by van der Wel et al. (2008). Briefly, GALFIT (Peng et al. 2002) is used to determine from the $r$ band the radii, axis ratios, position angles, and total magnitudes, assuming a de Vaucouleurs (1948) surface brightness profile. The smearing effect of the point-spread function is taken into account in the model. Most galaxies have sizes comparable to the point-spread function, that is, the global axis ratio is reliably recovered. While we use the de Vaucouleurs-derived values, we note that adopting surface brightness models with a free Sérsic (1968) index does not lead to a significantly different $b / a$ distribution. The systematic difference between the two values is only 0.004 in the median, the scatter is 0.075 , and for less than $1 \%$ of all galaxies the values differ by more than 0.20 .

The drawback of using the best-fitting axis ratio as a proxy for morphology is that its value may not be very meaningful in case a galaxy is a system with three components (say, bulge, 
disk, and bar) which all contribute significantly to the light. More detailed modeling of the surface brightness profiles may alleviate this problem. However, this would be an extensive study in itself and, moreover, prone to other, potentially more serious systematic errors due to the limited information in the SDSS images of the galaxies in our sample.

Stellar masses are estimated with a simple conversion from color to mass-to-light ratio: $M / L_{r}=1.097 \times(g-r)-0.306-$ 0.1 taken from Bell et al. (2003), where $L_{r}$ and $g-r$ are computed at $z=0$, and with a $0.1 \mathrm{dex}$ shift downward to normalize all stellar masses to the Kroupa (2001) stellar initial mass function. The assumed cosmology is $\left(\Omega_{\mathrm{M}}, \Omega_{\Lambda}, h\right)=$ $(0.3,0.7,0.7)$.

We match this sample with the galaxy group catalog constructed by Yang et al. (2007). Their method first uses a friendsof-friends algorithm to identify the centers of potential groups, whose characteristic luminosity is then estimated. Using an iterative approach, the adaptive group finder then uses the average mass-to-light ratios of groups, obtained from the previous iteration, to assign a tentative mass to each group. This mass is then used to estimate the mass of the underlying halo that hosts the group, which is in turn used to determine group membership in redshift space. Finally, each individual group is assigned a halo mass, estimated from the group's stellar mass, by ranking those stellar masses and the halo masses from a numerical simulation of cosmological structure growth. For more details, we refer the reader to Yang et al. (2007).

The group finder is optimized to maximize completeness while minimizing contamination by interlopers. The most massive galaxy of each group is, by definition, the "central galaxy," and is usually located near the geometric center of the group. The other galaxies in the group are "satellite" galaxies. However, the distinction between "central" and "satellite" galaxies is not of great importance for the our analysis.

As Yang et al. (2007) constructed the catalog from SDSS Data Release 4 (Adelman-McCarthy et al. 2006), we do not have complete information for all galaxies in our initial sample, which is selected from Data Release 6. After cross matching, we have a sample of $\sim 12,800$ quiescent galaxies that are more massive than $M_{*}=2.5 \times 10^{10} M_{\odot}$ (note that dwarf galaxies are not considered here), and which also have been assigned membership of a group as either a satellite or a central galaxy. This sample is used in this paper. A complementary sample of $\sim 31,000$ star-forming galaxies (i.e., those galaxies that do not satisfy the selection criteria for quiescence given above, and in the same redshift range, with known colors, stellar masses, and group masses) is used in this paper to quantify the fraction of quiescent galaxies in different environments, that is, in halos with different masses.

\section{A PHYSICAL DESCRIPTION OF THE MORPHOLOGY-DENSITY RELATION}

\subsection{Dark Matter Halo Mass and the Quiescent Galaxy Fraction}

In Figure 1, we show the fraction of $\operatorname{red}^{5}$ and quiescent galaxies as a function of halo mass. Essentially all quiescent galaxies are red, but the reverse is not the case. Late-type galaxies with less than average star formation activity may

\footnotetext{
5 Our definition of a red galaxy is a galaxy which is less than 2 standard deviations bluer than the center of the red sequence, i.e., galaxies that satisfy $g-r>0.07 \times \log \left(M_{*} / 10^{11} M_{\odot}\right)+0.75$, where $g-r$ is computed at $z=0$, are red.
}

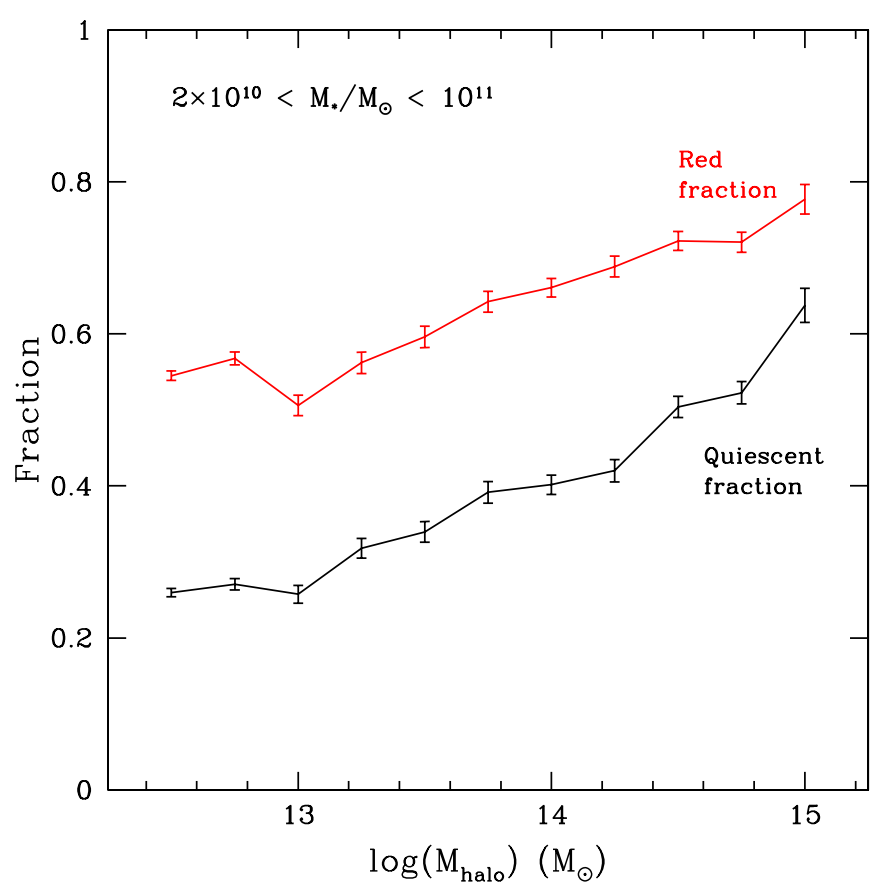

Figure 1. Fraction of spectroscopically selected, quiescent (black lines and error bars) and red (red lines and error bars) galaxies with mass $2.5 \times 10^{10}<$ $M_{*} / M_{\odot}<10^{11}$ as a function dark matter halo mass from the Yang et al. (2007) group catalog. The error bars indicate standard binomial errors in the fraction (these are henceforth used in all figures which show fractions). Both the quiescent and red fraction increase with halo mass, and it is apparent that "quiescence" is a more stringent criterion than "redness."

(A color version of this figure is available in the online journal.)

satisfy our color criterion, and edge-on spiral galaxies may be relatively red because of extinction. Moreover, galaxies with genuinely low star formation rates but with some level of nuclear activity will be included in the red sample, but not in the quiescent sample. Generally speaking, it is important to keep in mind that a sample of galaxies selected by optical color consists of galaxies with a wide range of properties and should not be equated with a sample of quiescent galaxies. We focus on quiescent galaxies for the pragmatic reason that it is well defined to address the question at hand: to what extent are environmental trends due to the loss of cold gas and the consequential decline in star formation activity?

Despite these complications, for both the red and the quiescent sample, the observed trend resembles the picture that was originally sketched by the MDR, that is, the fraction of red/quiescent galaxies increases with halo mass. However, no distinction between high- and low-mass galaxies is being made here. As a consequence, the observed trend includes, in addition to the actual relationship between galaxy properties and environment, if it exists, the relation between color/star formation activity and galaxy mass, and between galaxy mass and environment.

To gain a clearer perspective, we split the sample into galaxies in high-mass halos $\left(M_{\text {halo }}>2.5 \times 10^{14} M_{\odot}\right)$ and galaxies in lowmass halos $\left(M_{\text {halo }}<2.5 \times 10^{14} M_{\odot}\right)$. Our choice for this value, which corresponds to the mass of a relatively low-mass cluster with a velocity dispersion of $\sim 400-500 \mathrm{~km} \mathrm{~s}^{-1}$, to distinguish between high- and low-mass halos is motivated in Section 3.2. In Figure 2, we compare the fractions of quiescent galaxies at fixed galaxy mass in high- and low-mass halos. Now that we have taken out the galaxy mass dependence, and, to first order, the halo mass dependence, the intrinsic environmental dependence 


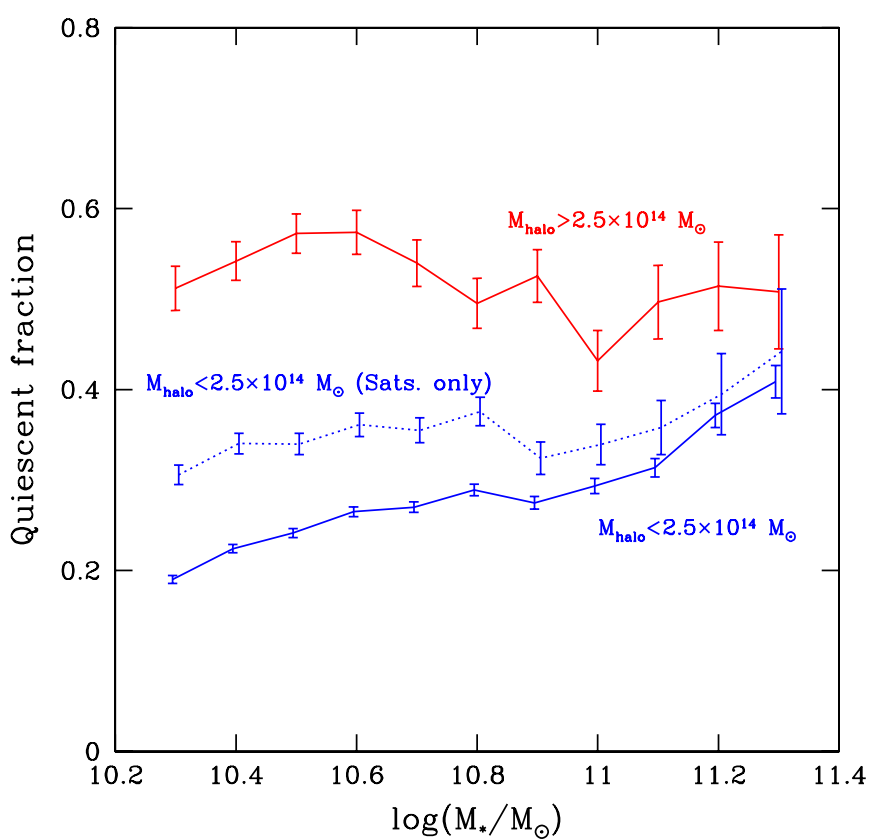

Figure 2. Fraction of quiescent galaxies as a function of galaxy stellar mass The red line indicates galaxies in high-mass halos (these are essentially all satellite galaxies); the solid blue lines indicate galaxies in low-mass halos; the dotted blue line shows satellite galaxies in low-mass halos. The quiescent galaxy fraction is significantly higher in high-mass halos than in low-mass halos, even at fixed galaxy stellar mass. This is partially, but not mostly, due to the difference between central and satellite galaxies: satellites in high-mass halos are different from satellites in low-mass halos.

(A color version of this figure is available in the online journal.)

of star formation activity is revealed. The same trend, but then for color instead of star formation activity, was found before by Weinmann et al. (2006), justifying the not entirely obvious assumption that optical color can be used to directly trace star formation activity. The trend seen in Figure 2 is partially driven by the different properties of central and satellite galaxies (e.g., van den Bosch et al. 2008; Pasquali et al. 2009; Skibba 2009), but this is not the dominant factor. Also for satellite galaxies, we see that those in high-mass halos are more frequently quiescent than in low-mass halos. This can be seen in Figure 2, where the fraction of quiescent satellites is smaller in low-mass halos than in high-mass halos. We note that essentially all galaxies in high-mass halos are satellites.

\subsection{High-mass Halos and Quiescent Galaxies with Prominent Disks}

Now that we have established that galaxies in high-mass halos are more often quiescent than galaxies in low-mass halos, we address the question what type of galaxy drives this trend. We use our axis ratio $(b / a)$ measurements as a tracer of the frequency of disks in the population of quiescent galaxies. Hence, we can test the hypothesis that the truncation of star formation in high-mass halos is associated with the destruction, preservation, or production of thin, stellar disks. Axis ratios do not provide a very sensitive test, because of projection effects, but they are very robust, with negligible systematic and random measurement errors (see Section 2).

In Figure 3 (left), we show the $b / a$ distribution for quiescent galaxies in a narrow range of stellar mass, as a function of halo mass. The halo mass range shown here, $M_{\text {halo }}=10^{13}-10^{15} M_{\odot}$, probes a large range of environments, from groups with a few $L^{*}$ galaxies to large clusters with hundreds of $L^{*}$ galaxies and velocity dispersions of $\sim 1000 \mathrm{~km} \mathrm{~s}^{-1}$, such as the Coma cluster. At halo masses $M_{\text {halo }}<10^{13} M_{\odot}$ the group catalog is essentially a catalog of relatively isolated galaxies, the halos of which have been assigned masses that correspond directly to the masses of those galaxies. We omit this mass range from the figure because it would not show trends with environment/halo mass, but rather the relationship between galaxy mass and axis ratio.

Figure 3 reveals that high-mass halos host a larger fraction of quiescent galaxies with small $b / a$ than low-mass halos, which are dominated by relatively round systems. In particular for galaxies with masses ranging from $M_{*}=5 \times 10^{10} M_{\odot}$ to $M_{*}=10^{11} M_{\odot}$ the trend is highly significant (see Figure 3, right). In this galaxy mass range, a transition in the $b / a$ distribution occurs around $M_{\text {halo }}=2.5 \times 10^{14} M_{\odot}$, the mass of a relatively small cluster, which explains our choice to distinguish between high- and low-mass halos at this particular value, first introduced in Section 3.1, and used throughout this paper.

Very few high-mass galaxies have pronounced disks, as shown recently by van der Wel et al. (2009). Massive galaxies in high-mass halos do not form an exception to this general rule, and, as a result, the axis ratio distribution is not seen to vary with halo mass.

Because the majority of galaxies with relatively small masses $\left(M_{*}<5 \times 10^{10} M_{\odot}\right)$ have disks, it is harder to distinguish an excess population of disks in high-mass halos. A small difference, however, is still apparent, as can be seen in Figure 3 (right). The relatively small effect on the axis ratio distribution does not imply that environmental processes are weaker at these lower masses; on the contrary, environmental processes are more pronounced at lower masses, as can be seen in Figure 2.

In order to formalize the trends seen in Figure 3, we analyze the cumulative $b / a$ distributions for different ranges in galaxy mass. These are shown in Figure 4. As was already indicated above, the $b / a$ distributions of massive galaxies in high- and low-mass halos are statistically indistinguishable. For galaxies with masses below $M_{*}=10^{11} M_{\odot}$, however, there is a notable and significant difference. According to the standard Kolmogorov-Smirnov test it is highly unlikely that quiescent galaxies have the same $b / a$ distributions in low- and high-mass halos, in the sense that high-mass halos more frequently host disky quiescent galaxies (see Figure 4).

Recently, van den Bergh (2009) attempted to identify such a correlation between flatness and environment, but did not find a statistically significant trend. A sample of several hundred galaxies is perhaps insufficient to disentangle the large variety of underlying correlations between global properties of galaxies. The sample that we use in this paper is almost 2 orders of magnitude larger, which adequately remedies this problem.

\subsection{Disky Quiescent Galaxies Drive the Morphology-Density Relation}

In Section 3.1, we established that high-mass halos have a higher fraction of quiescent galaxies-at a given galaxy mass-than low-mass halos (see Figure 2). In Section 3.2, we found that quiescent galaxies in high-mass halos are more frequently disky than in low-mass halos (see Figures 3 and 4). These two observations could be physically related in which case the increased quiescent fraction in high-mass halos is directly caused by an increased fraction of quiescent disk galaxies; see also the discussion by van den Bosch et al. (2008).

We test this hypothesis with a simple model. The idea is that the quiescent population in high-mass halos consists of galaxies that would be quiescent even if they had been located in less 

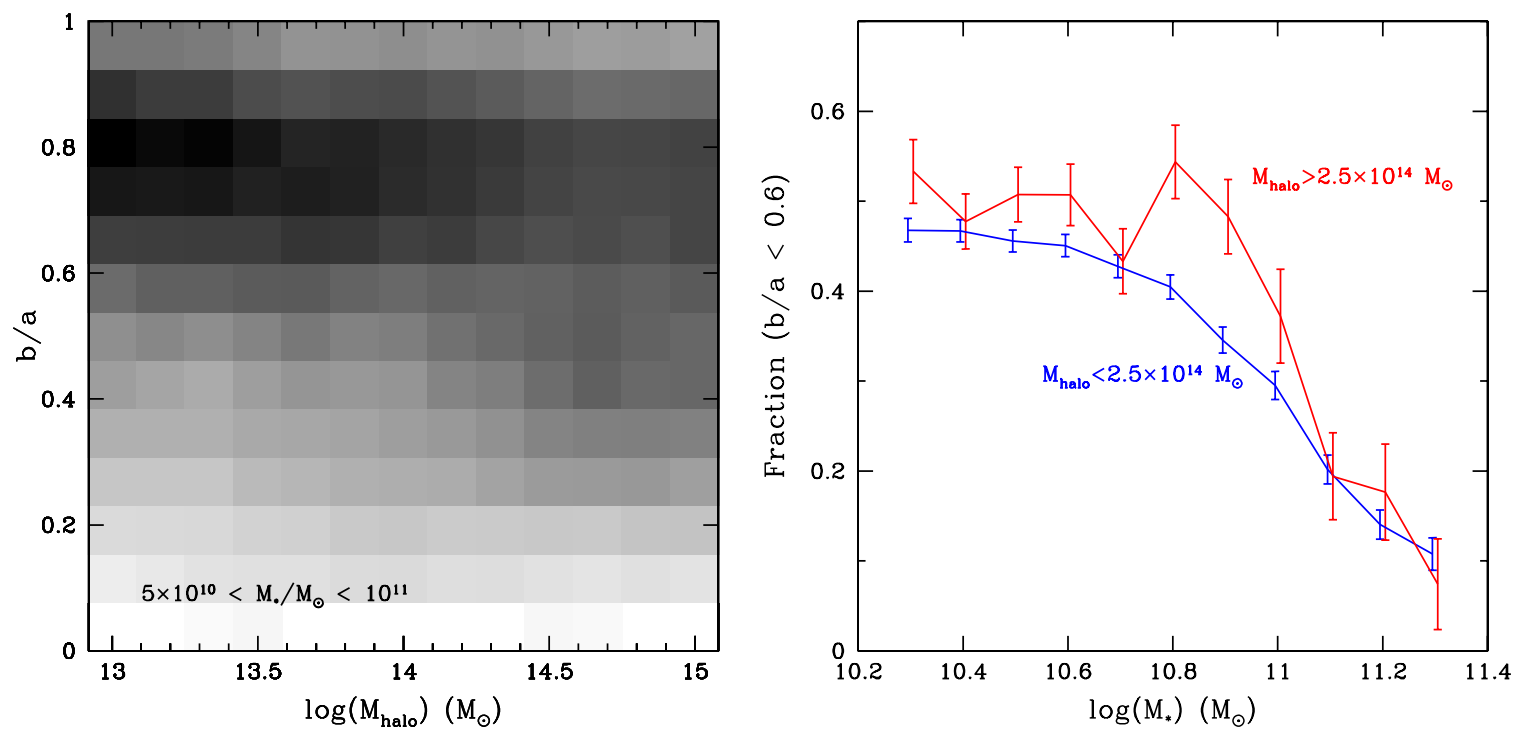

Figure 3. Left: axis ratio $(b / a)$ distribution as a function of dark matter host halo mass for quiescent galaxies in the mass range $5 \times 10^{10}<M_{*} / M_{\odot}<10^{11}$. The gray scale indicates the frequency of $b / a$, where we normalize to unity the total number of galaxies in each stellar mass bin, the width of which is indicated by the grid size. Smoothing in both directions is applied to reduce the effects of the shot noise. The presence of the dark region near the top left corner implies that quiescent galaxies in low-mass halos are typically round. The $b / a$ distribution at high halo masses is more uniform, with a higher fraction of elongated, that is, disky, quiescent galaxies. Right: fraction of quiescent galaxies with axis ratios $b / a<0.6$ as a function of galaxy mass. The red line represents galaxies in massive halos; the blue line represents galaxies in low-mass halos. Error bars represent the Poisson uncertainty. Quiescent galaxies with $M_{*} \lesssim 10^{11} M_{\odot}$ in high-mass halos are significantly more often elongated, that is, disk dominated, than their counterparts in low-mass halos.

(A color version of this figure is available in the online journal.)

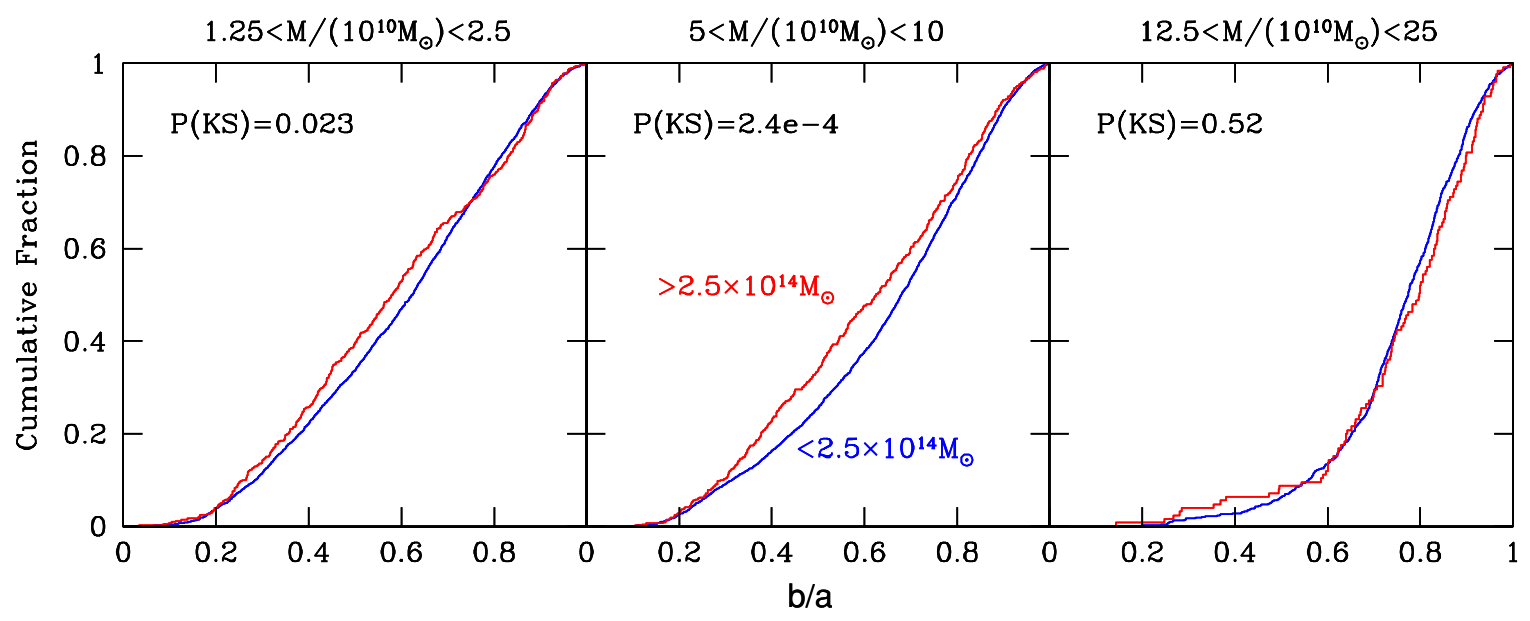

Figure 4. Cumulative axis ratio distribution for quiescent galaxies in three different galaxy mass bins. The red lines reflect the $b / a$ distribution of galaxies in high-mass halos; the blue lines indicate the $b / a$ distribution of galaxies in low-mass halos, as labeled in the middle panel. $P(\mathrm{KS})$ is the probability that the $b / a$ values of galaxies in low- and high-mass halos are drawn from the same distribution, using the standard Kolmogorov-Smirnov test. For high-mass galaxies (right) the two $b / a$ distributions are statistically indistinguishable. For galaxies with masses $M_{*}<10^{11} M_{\odot}$, the probability that the $b / a$ distributions in high-and low-mass halos are the same is very small: high-mass halos contain an excess population of disk-dominated quiescent galaxies compared to low-mass halos.

(A color version of this figure is available in the online journal.)

massive halos, that is, regardless of their environment, and an additional population that is quiescent as a direct consequence of their environment. We assume that the latter population is the result of gas stripping, which leaves stellar disks intact and does not alter the structural properties of galaxies. We discuss this assumption below.

The expected $b / a$ distribution of quiescent galaxies in highmass halos is inferred by adding these two populations: (1) a population with the $b / a$ distribution of similarly massive quiescent galaxies in low-mass halos, which represent galaxies that are quiescent regardless of halo mass and (2) a population of galaxies, projected along random lines of sight, with the same intrinsic axis ratios as similarly massive spiral galaxies (i.e., non-quiescent $L^{*}$ galaxies), which represent galaxies that are quiescent because of their environment. According to Padilla \& Strauss (2008), $L^{*}$ spiral galaxies have intrinsic axis ratios that are normally distributed, with a mean of 0.26 and a scatter of 0.06 . The motivation for this choice is that such spiral galaxies will, upon entering a massive halo, eventually cease to form stars without changes in structure.

Besides the $b / a$ distributions of the two populations in the model, we also have to choose the relative numbers of galaxies in the two populations. We choose these relative numbers such that we precisely match the increase in the quiescent galaxy fraction from low- to high-mass halos, which we described in Section 3.1 and showed in Figure 2. Hence, if our gas-stripping hypothesis 

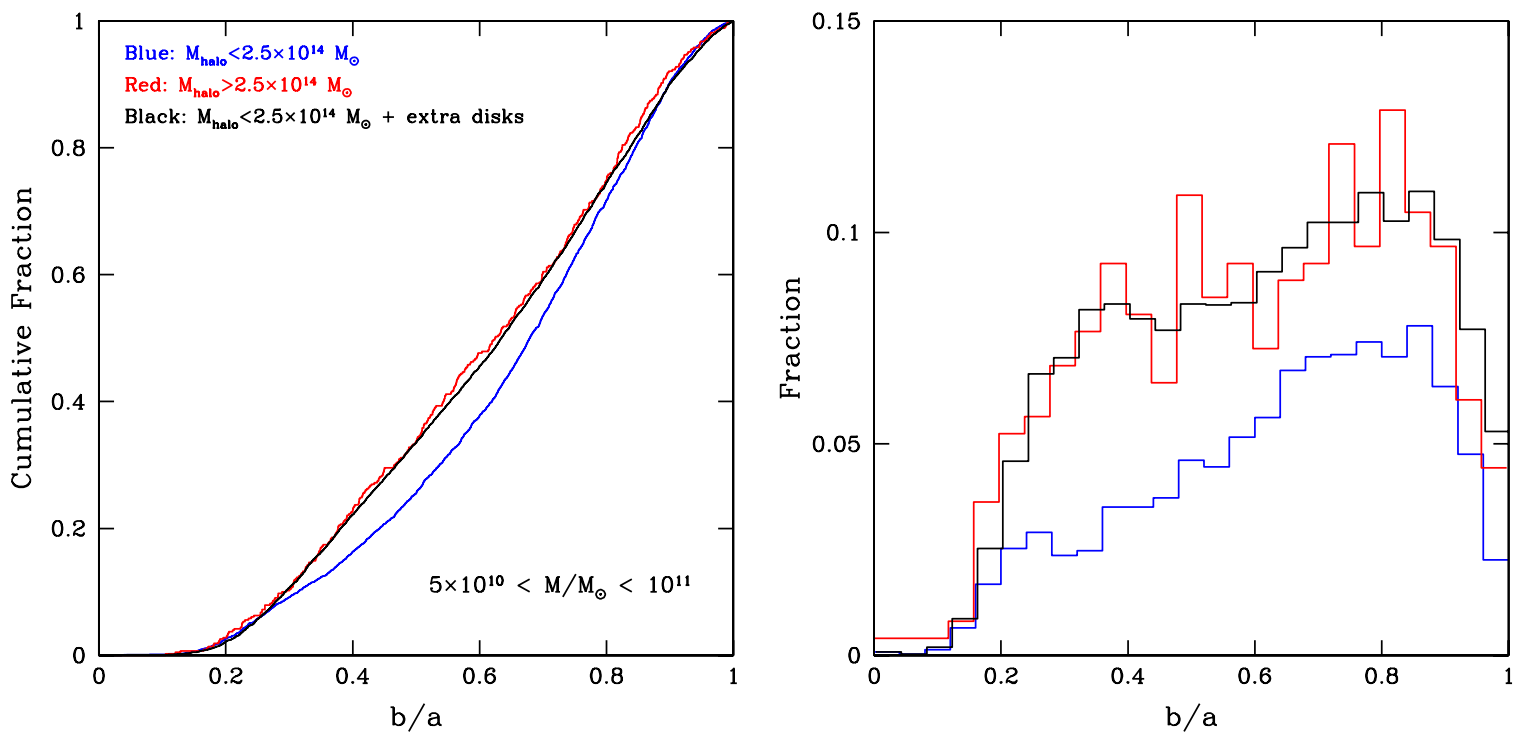

Figure 5. Cumulative distribution (left) and histogram (right) of axis ratios of quiescent galaxies with masses in the range $5 \times 10^{10}<M_{*} / M_{\odot}<10^{11}$. The solid red lines reflect the $b / a$ distribution of galaxies in high-mass halos; the blue lines reflect the $b / a$ distribution of galaxies in low-mass halos. The black line is the $b / a$ distribution in low-mass halos (i.e., the blue line) augmented by a population of "extra" quiescent disk galaxies. The latter is generated by projecting along random lines of sight a population of disk galaxies with the same intrinsic axis ratio distribution as $L^{*}$ spiral galaxies (see the text for details). The relative number of these extra disk galaxies is chosen such that their addition makes up for the difference in the quiescent galaxy fractions in high- and low-mass halos (see Figure 2) for the galaxy stellar mass range considered here. These extra disk galaxies can be thought of as galaxies whose star formation has been truncated as a results of some environmental process that leaves the stellar disk intact. The resulting model $b / a$ distribution is statistically indistinguishable from the observed $b / a$ distribution in high-mass halos: the Kolmogorov-Smirnov test yields a probability of 0.83 that the distributions are the same. Therefore, the increased fraction of quiescent galaxies in high-mass halos seen in Figure 2 is likely caused by the increased fraction of disky, quiescent galaxies seen in Figures 3 and 4.

is correct, then the model $b / a$ distribution is consistent with the observed $b / a$ distribution in high-mass halos.

In Figure 5, we compare the predicted and observed $b / a$ distributions in high-mass halos for galaxies in the mass range $5 \times 10^{10}<M_{*} / M_{\odot}<10^{11}$. The population of "extra," disky quiescent galaxies added to the $b / a$ distribution of galaxies in low-mass halos changes the $b / a$ distribution in such a manner that the $b / a$ distribution in high-mass halos is accurately reproduced. There is no significant difference between the predicted and observed $b / a$ distributions in high-mass halos: according to the Kolmogorov-Smirnov test, the probability that the distributions are the same is $0.83 \pm 0.06 .^{6}$ This is to be compared with the low probability $\left(2.3 \times 10^{-4}\right)$ that the $b / a$ distributions in low- and high-mass halos are the same, a remarkable success for such a simple model.

We repeat the exercise for galaxies with lower masses (the left-hand panel in Figure 4). The difference between low- and high-mass halos is less pronounced and less significant here. Yet, adding an extra population of faded spirals to the $b / a$ distribution observed in low-mass halos, as described above, we again reproduce with good confidence the observed $b / a$ distribution in high-mass halos.

This model, however, does not work for high-mass galaxies $\left(M>10^{11} M_{\odot}\right)$. In the right-hand panel of Figure 4 can be seen that the $b / a$ distributions of massive galaxies are not different in low- and high-mass halos, yet, Figure 2 shows that even for such massive galaxies there is a small, but significant difference in the quiescent fraction. As a consequence, modeling the additional

\footnotetext{
6 In a Monte Carlo simulation, this is the average of 1000 realizations of the random inclination distribution of the "extra" disky, quiescent galaxy population. This produces a scatter of 0.06 in the inferred probability that the
} distributions are the same. quiescent galaxies in high-mass halos with a population of faded spiral galaxies does not reproduce the observed $b / a$ distribution in high-mass halos. We discuss this further in Section 4.

We note that none of the above results change if we restrict the analysis to satellite galaxies alone. The increased fraction of quiescent satellite galaxies in high-mass halos (see Figure 2) is well described by an increased number of quiescent, diskdominated galaxies, analogous to our above description of the entire population of quiescent galaxies.

The assumption in all of the above is that the axis ratio does not change when a star-forming spiral galaxy transforms into a quiescent, disky galaxy. More explicitly, we assume that the stellar mass distribution is not significantly affected by the process that truncates the star formation. However, it is good to keep in mind that tidal interactions may thicken the disk, and radial mixing, on the long term, can change the bulge-to-disk ratio (see Boselli \& Gavazzi 2006, for an overview). We also neglect changes in surface brightness profiles due to changes in mass-to-light ratio gradients caused by either stellar population effects or rearrangement of dust. However, the fact that we can model the axis ratio distribution in high-mass halos by assuming that the structure of a galaxy does not change when its star formation activity is truncated, suggests that our assumption is reasonable.

This section presents the central result of this study. In summary, we connect the increased fraction of quiescent galaxies in high-mass halos (see Figure 2) to the increased fraction of disk-dominated, quiescent galaxies in high-mass halos (see Figures 3 and 4). We demonstrate quantitatively that the increased fraction of quiescent galaxies in high-mass halos is in fact driven by the population of disk-dominated galaxies (Figure 5). In the following section, we discuss the ramifications of these findings in the context of the historical discussion of the MDR and in light of other recent results. 


\section{DISCUSSION}

\subsection{The Definition of "Morphology" and Its Interpretation}

The traditional morphological classification of galaxies is based on two observable properties: smoothness of the light distribution and the presence/absence of a substantial disk (e.g., Sandage 1961). Physically speaking, smoothness reflects star formation activity, whereas the presence of a disk implies a dynamically cold component. Because these properties are correlated with each other, and with a host of other galaxy parameters, morphology is not a fundamental galaxy property, which renders morphological studies difficult to interpret. We refer to Blanton \& Moustakas (2009) for a recent, general overview of the field.

In any case, these two observables, smoothness, and diskiness, divide the general population into three broad classes: ellipticals (smooth, negligible disks), lenticulars or S0s (smooth, significant disks), and spirals (not smooth, significant disks). ${ }^{7}$ Smoothness is related to the lack of star formation activity; hence, our selection of quiescent galaxies is equivalent to selecting ellipticals and S0s, that is, early-type galaxies.

From the early SDSS studies by, for example, Kauffmann et al. (2003) and Hogg et al. (2004), we learned that diskiness is primarily related to galaxy mass, and also that its correlation with environment is weak (Kauffmann et al. 2004; Blanton et al. 2005). Smoothness, on the other hand, has been demonstrated to correlate with environment as well (van der Wel 2008), which should be interpreted as an environmental dependence of star formation activity (see also Lewis et al. 2002; Gómez et al. 2003; Balogh et al. 2004; Tanaka et al. 2004; Weinmann et al. 2006; Baldry et al. 2006; Park et al. 2007, who all find a correlation between color/star formation and environment). Hence, we have that the two physical properties that make up morphology behave differently as a function of fundamental galaxy properties, reconciling the result that whereas morphology is sensitive to environmental factors, structure is not, or at least less so (van der Wel 2008; Bamford et al. 2009; Skibba et al. 2009).

\subsection{Gas Stripping and the Morphology-Density Relation}

The degree to which the star formation activity of a galaxy is environmentally affected depends on halo mass (Weinmann et al. 2009). Satellite galaxies in high-mass halos have different color profiles and are generally redder than their equally massive counterparts in low-mass halos. Model constraints on the amount of time that galaxies have spent as satellites and the observed correlation between halo mass and satellite color imply that the decrease in star formation activity is gradual, taking place on a time scale of several Gyr. This is reinforced by the dependence of star formation activity of satellite galaxies in massive halos on cluster-centric distance (von der Linden et al. 2009), and, even more generally, by the non-zero fraction of blue satellite galaxies (Kang \& van den Bosch 2008; Font et al. 2008; Skibba \& Sheth 2009).

These results are inconsistent with a scenario in which the entire interstellar medium is rapidly stripped as a result of ram pressure. Increasingly sophisticated simulations indicate that the time scale for gas loss through ram-pressure stripping is longer than expected on the basis of simple, analytical estimates (e.g., Roediger \& Brüggen 2007). The picture that is emerging is

\footnotetext{
7 We are only considering galaxies with masses more than $20 \%$ of that of an $L^{*}$ star galaxy, that is, the following discussion does not apply to dwarf galaxies, which constitute a somewhat separate class of objects.
}

that star formation continues after infall, but gradually declines as the fuel for star formation is removed relatively slowly and gently by the stripping of hot gas outside the cold disk or atomic hydrogen in the outer parts (e.g., Koopmann \& Kenney 2004). However, we note that the physical process at work here is still ram-pressure stripping, but that this only efficiently acts on part of the interstellar medium.

Explicit evidence for this process is provided by the observation of an excess of red, late-type galaxies in the outskirts of a massive cluster (Wolf et al. 2009; Gallazzi et al. 2009), which have lower star formation rates than late-type galaxies in the general field. More generally, the clustering properties of red spiral galaxies imply that such galaxies are often satellite galaxies in massive halos (Skibba et al. 2009). Interestingly, such "anemic" spirals were identified and discussed early on, by van den Bergh (1976) and have been suggested as an explanation for the MDR (Koopmann \& Kenney 1998).

The end result of the gas-stripping process, that is, the descendant of a red spiral galaxy with a declining star formation rate, is a quiescent, disk-dominated galaxy of which the bulk of the stellar population is at least several Gyr old, in agreement with spectroscopically inferred age estimates of quiescent galaxies in general (e.g., Gallazzi et al. 2005; Graves et al. 2009a). These conclusions, together with the dependence on halo mass of the average amount of time that a galaxy has spent as a satellite, may explain the correlation between the stellar population ages of red galaxies and environment (Cooper et al. 2010).

Our results provide strong evidence for the presence of such "starved", disky galaxies in massive halos. Moreover, our results imply that these galaxies represent the increased fraction of quiescent galaxies in high-mass halos. Hence, our results, combined with those discussed above, imply that a gradual removal of the gaseous interstellar medium of spiral galaxies is responsible for the MDR. This conclusion is physically motivated by the observed decreased star formation in satellite galaxies and the time scale associated with this phenomenon (e.g., Weinmann et al. 2009), and quantitatively matched by the observed increase in the fraction of disk-dominated, quiescent galaxies in massive halos (this paper).

\subsection{Are SO Galaxies Stripped Spiral Galaxies?}

In this paper, we argue that slow gas stripping through interaction with the intergalactic medium causes spiral galaxies to stop forming stars and eventually turn in disky, quiescent objects, that is, S0 galaxies. Furthermore, we claim that this explains the existence of the MDR for galaxies with masses $M>2.5 \times 10^{10} M_{\odot}$. Note that in this picture, galaxies do not undergo structural changes, in the sense that their bulge-to-disk ratios stay the same.

We stress that we do not claim that all S0 galaxies formed through this process. The existence of S0 galaxies and existence the MDR do not necessarily have the same explanation, which was already noted by Postman \& Geller (1984). Only 15\% of the galaxies in our sample are located in dark matter halos with masses $M_{\text {halo }}>2.5 \times 10^{14} M_{\odot}$, where the increased presence of quiescent disk galaxies is noticeable. For this sub-population, the fraction of quiescent galaxies is 1.5-2 times higher than in lower-mass halos (see Figure 2), implying that only 5\%-10\% of all quiescent galaxies are quiescent as a result of differential environmental effects, by which we mean those environmental effects that do not or less efficiently act in lower-mass halos. Although these are rough estimates, it is safe to conclude that the process that is responsible for the MDR is not the dominant 
contributor to the truncation of star formation and the formation of the red sequence in general.

It is not surprising, then, that the MDR is as weak as it is: morphological fractions change only by a factor 2 over many orders of magnitude in environmental density, as was pointed out before, by, e.g., Dressler (2004). The star formation and structural properties of galaxies depend much more strongly on internal galaxy properties (mass, velocity dispersion, and surface mass density) than on their environment. Many central galaxies are quiescent and many quiescent satellite galaxies had their star formation truncated before their becoming satellites (van den Bosch et al. 2008).

Although low-mass satellites in low-mass halos could still be the result of gas stripping, it seems unavoidable that other mechanisms that truncate star formation are important. Merging may explain the cessation of star formation by means of gas exhaustion through enhanced star formation activity during the merger phase. Stellar disks are not destroyed in the case of minor merging, although the bulge-to-disk ratio can increase (e.g., Bekki 1998; Naab et al. 1999), and a sequence of minor mergers can result in an elliptical galaxy (Bournaud et al. 2007). It is generally accepted that major merging results in round remnants (Toomre \& Toomre 1972; Barnes \& Hernquist 1996), although such events can also lead to the formation of flattened, rotating systems, especially if the progenitors are relatively gas rich (Cox et al. 2006).

Whether merging will turn out to be the main mechanism to produce quiescent galaxies remains to be seen. However, quiescence seems to be related to the presence of a bulge (e.g., Bell 2008), produced by merging or otherwise. In its barest form, this relationship manifests itself through the correlation between structure and star formation activity. Hence, it is not at all surprising that S0s, which are quiescent, have larger bulges than star-forming spirals. In other words, S0s are not, generally speaking, gas-stripped spirals. However, as explained above, this is not at odds with our claim that gas-stripping results in the existence of the MDR.

If merging is an important driver of bulge growth, then differences between S0s and spirals are expected. These differences include the offset in the Tully \& Fisher (1977) relation between spirals and S0s (M. J. Williams et al. 2010, in preparation), the prominence of thick disks in S0s compared to spirals (e.g., Burstein 1979), and the lower bar fraction for S0s (Aguerri et al. 2009; Laurikainen et al. 2009). The accretion of satellite galaxies/minor merging can destroy bars and cause thick disks to grow more prominent, either through heating up the pre-existing thin disk (e.g., Quinn et al. 1993) or by depositing tidally stripped debris from the accreted systems at large scale heights (e.g., Gilmore et al. 2002; Martin et al. 2004). In addition, minor accretion events in the absence of gas is expected to lead to a thicker disk than in the presence of gas (e.g., Moster et al. 2009). Hence, even similar satellite accretion histories for S0 and spiral galaxies can lead to differences between the thick disk components, and the general prominence of the surviving disk.

In summary, the global differences between S0 and spiral galaxies do not argue against slow gas stripping as an explanation for the MDR. Most quiescent galaxies, including those with prominent disks, are not the result of differential environmental processes, that is, processes that do not act (efficiently) in low-density environments/low-mass halos. If environmental processes are important, they act efficiently in all environments. However, it seems inescapable to conclude that other truncation mechanisms, likely associated with merging, are important, because bulge growth and the truncation of star formation go hand in hand.

\subsection{Elliptical Galaxies and Environment}

Because the increase in the quiescent galaxy fraction with halo mass can be fully explained by the increased fraction of intrinsically flattened galaxies (Section 3.2), it follows that the fraction of intrinsically round quiescent galaxies (elliptical galaxies) does not change with halo mass, at least, for galaxy masses below $10^{11} M_{\odot}$. At first sight, this seems to be at odds with the increased fraction of elliptical galaxies, with respect to the total population, at high local density (Dressler 1980).

Whitmore et al. (1993) showed that galaxy morphology varies strongly with position within a cluster. They argue that the result from Dressler can be explained by a higher fraction of elliptical galaxies in the cluster core (within the central $0.25 \mathrm{Mpc}$ ) than elsewhere. Using the distance-to-group-center estimates from Yang et al. (2007), we check whether we can reproduce the trend shown by Whitmore et al. (1993). In order to roughly match the properties of the galaxies in the sample used by Dressler (1980) and Whitmore et al. (1993), we select galaxies from our sample with mass $M_{*}>6 \times 10^{10} M_{\odot}$ in groups with mass $M_{\text {halo }}>2.5 \times 10^{14} M_{\odot}$. Quiescent galaxies in that sample within the central $0.25 \mathrm{Mpc}$ of the centers of their respective groups are significantly rounder than galaxies at larger distances from the group centers, which implies that elliptical galaxies are more prevalent than S0s in cluster cores, and that our sample shows the same trend as identified by Whitmore et al. (1993).

It is important to note that the centers of groups and clusters tend to be populated by the most massive galaxies. This is relevant for the present discussion because morphological type depends strongly on galaxy mass: the axis ratio distribution implies that essentially all high-mass galaxies are intrinsically round (van der Wel et al. 2009), that is, those are all elliptical galaxies. At lower masses, many galaxies have significant disks (see also Figure 4). This suggests that the increased fraction of ellipticals in group centers includes a contribution from two underlying trends: group centers host more massive galaxies, which are, in turn, more often ellipticals. We find that this fully explains the dependence of the elliptical fraction on distance to the group center: the axis ratio distribution of quiescent galaxies with a given mass does not change with distance to group center.

Recently, von der Linden et al. (2009) showed that satellite galaxies in massive groups do not show a correlation between mass and distance to the group center. We find the same, and we only find mass segregation if the central galaxies are included. The implication is that the Whitmore et al. (1993) result that elliptical galaxy fraction increases toward the group center can be fully understood by distinguishing between central and satellite galaxies.

In summary, the increased fraction of elliptical galaxies in dense environments (Dressler 1980) and cluster cores (Whitmore et al. 1993) is not at odds with our claim that slow gas stripping of infalling spiral galaxies explains the MDR at fixed galaxy mass. The increased elliptical galaxy fraction in galaxy cores is simply the consequence of high-mass, elliptical galaxies preferring the inner regions. We show that, at fixed galaxy mass, S0 galaxies are more prevalent than ellipticals in clusters compared to lower-density environments, an issue that was not discussed in the early, seminal works by, e.g., Dressler (1980) and Postman \& Geller (1984). 


\subsection{The Morphological Mix of High-redshift Clusters of Galaxies}

With the arrival of the Hubble Space Telescope, it became possible to study the morphologies of galaxies in distant clusters (Dressler et al. 1997; Fasano et al. 2000; Treu et al. 2003; Postman 2005; Smith et al. 2005), and establish that the Hubble sequence and the MDR were already in place at $z \sim 1$. The same is the case for the underlying physical correlations between star formation activity and environment (e.g., Cooper et al. 2007; Patel et al. 2009; Tran et al. 2009).

There are two reasons to suspect that the morphological mix may change with look-back time. First, as cluster halos continue to assemble hierarchically through the accretion of smaller halos, newly infalling galaxies are added to the cluster population. This infall and merging process is directly observed at all redshifts, and must be an ongoing process (e.g., Burns et al. 1994; Markevitch et al. 2002). This process adds galaxies with field-like properties, which may differ from the already present cluster galaxies. Second, at higher redshift, the typical star formation rate is higher for spiral galaxies (Lilly et al. 1996; Madau et al. 1996), which suggests that the morphological mix may change with look-back time.

Indeed, the fraction of spiral/star-forming galaxies is observed to increase with redshift (e.g., Dressler et al. 1997; Smith et al. 2005; Postman 2005; Poggianti et al. 2006; Simard et al. 2009). In general, these studies suggest that the population of S0 galaxies is rapidly built up over the last $8 \mathrm{Gyr}$ from infalling spiral systems (Dressler et al. 1997; Postman 2005; Smith et al. 2005). It was shown by Holden et al. (2007) and van der Wel et al. (2007) that this is mainly due to the higher luminosity of spiral galaxies in distant clusters compared to those in local clusters: in mass-selected samples, little change is seen and the deficit of S0 systems appears to be overestimated (Holden et al. 2009).

The key result of van der Wel et al. (2007) is that, although there is little evolution in the fraction of E+S0 galaxies at the stellar masses of $L^{*}$ galaxies (cf. Bundy et al. 2009), there is still a strong relation between morphology and the local environment (see also Tasca et al. 2009). The persistence of this trend to higher redshifts implies that galaxies that become satellites in more massive halos transform from actively star-forming to quiescent galaxies. Such transformation are directly observed at intermediate redshifts (Moran et al. 2007).

Our result shows that there is a population of galaxies in high-mass halos that have a similar structure to star-forming field spiral galaxies, but lack the star formation. van der Wel et al. (2007) show that this population does not quickly appears at some given epoch. From this, we conclude that the assembly of the cluster population and the evolution of its star-forming properties are most naturally explained by the gradual stripping of the interstellar medium.

\section{SUMMARY AND CONCLUSIONS}

We use stellar masses, star formation activity, axis ratios, and group halo masses of galaxies in the SDSS to provide a physical interpretation of the morphology-density relation and its origin. Our findings are as follows.

1. The fraction of galaxies with low specific star formation rates (quiescent galaxies) increases with halo mass. This also holds at fixed galaxy mass and for satellite galaxies (Section 3.1).
2. Quiescent galaxies in high-mass dark matter halos ( $M_{\text {halo }}>$ $2.5 \times 10^{14} M_{\odot}$ ) are significantly more often disk dominated than quiescent galaxies in lower-mass halos (Section 3.2).

3. This additional population of disk-dominated quiescent galaxies quantitatively matches the increased fraction of quiescent galaxies in high-mass halos (Section 3.3).

Hence, our findings show that the morphology-density relation arises as a result of the increased fraction of disk-dominated, quiescent galaxies in high-mass halos, at the expense of diskdominated, star-forming galaxies, which are more frequently found in low-mass halos. Other studies (see Section 4.2) provide evidence that slow stripping of the interstellar medium is the most likely explanation for the decreased star formation activity of spiral galaxies in massive groups. We conclude that the slow stripping of gas from spiral galaxies, which does not strongly alter its structural properties of a galaxy, likely explains the MDR.

These findings are discussed in the context of the rich history of studies on galaxy morphologies and their environmental dependence (Section 4). In particular, we demonstrate that our conclusions are not incompatible with the suite of evidence that S0 galaxies and spiral galaxies have systematically different properties. Such evidence has often, and correctly, been invoked to argue that $\mathrm{S} 0$ galaxies cannot, generally speaking, be stripped spiral galaxies. Rather, the stripped, quiescent galaxies that drive the morphology-density relation are only a small subset of the entire population quiescent galaxies with disks.

We thank Frank van den Bosch and Anna Gallazzi for useful comments on the manuscript. Funding for the SDSS and SDSS-II has been provided by the Alfred P. Sloan Foundation, the Participating Institutions, the National Science Foundation, the U.S. Department of Energy, the National Aeronautics and Space Administration, the Japanese Monbukagakusho, the Max Planck Society, and the Higher Education Funding Council for England. The SDSS Web site is http://www.sdss.org/. The SDSS is managed by the Astrophysical Research Consortium for the Participating Institutions. The Participating Institutions are the American Museum of Natural History, Astrophysical Institute Potsdam, University of Basel, University of Cambridge, Case Western Reserve University, University of Chicago, Drexel University, Fermilab, the Institute for Advanced Study, the Japan Participation Group, Johns Hopkins University, the Joint Institute for Nuclear Astrophysics, the Kavli Institute for Particle Astrophysics and Cosmology, the Korean Scientist Group, the Chinese Academy of Sciences (LAMOST), Los Alamos National Laboratory, the Max-Planck-Institute for Astronomy (MPIA), the Max-Planck-Institute for Astrophysics (MPA), New Mexico State University, Ohio State University, University of Pittsburgh, University of Portsmouth, Princeton University, the United States Naval Observatory, and the University of Washington.

\section{REFERENCES}

Adelman-McCarthy, J. K., et al. 2006, ApJS, 162, 38

Adelman-McCarthy, J. K., et al. 2008, ApJS, 175, 297

Aguerri, J. A. L., Méndez-Abreu, J., \& Corsini, E. M. 2009, A\&A, 495, 491

Baldry, I. K., Balogh, M. L., Bower, R. G., Glazebrook, K., Nichol, R. C., Bamford, S. P., \& Budavari, T. 2006, MNRAS, 373, 469

Balogh, M. L., Baldry, I. K., Nichol, R., Miller, C., Bower, R., \& Glazebrook, K. 2004, ApJ, 615, L101

Balogh, M. L., Schade, D., Morris, S. L., Yee, H. K. C., Carlberg, R. G., \& Ellingson, E. 1998, ApJ, 504, L75

Bamford, S. P., et al. 2009, MNRAS, 393, 1324 
Barnes, J. E., \& Hernquist, L. 1996, ApJ, 471, 115

Bedregal, A. G., Aragón-Salamanca, A., \& Merrifield, M. R. 2006, MNRAS, 373,1125

Bekki, K. 1998, ApJ, 502, L133

Bekki, K., Couch, W. J., \& Shioya, Y. 2002, ApJ, 577, 651

Bell, E. F. 2008, ApJ, 682, 355

Bell, E. F., McIntosh, D. H., Katz, N., \& Weinberg, M. D. 2003, ApJS, 149, 289

Blanton, M. R., \& Berlind, A. A. 2007, ApJ, 664, 791

Blanton, M. R., Eisenstein, D., Hogg, D. W., Schlegel, D. J., \& Brinkmann, J. 2005, ApJ, 629, 143

Blanton, M. R., \& Moustakas, J. 2009, ARA\&A, 47, 159

Boselli, A., \& Gavazzi, G. 2006, PASP, 118, 517

Bournaud, F., Jog, C. J., \& Combes, F. 2007, A\&A, 476, 1179

Bundy, K., et al. 2009, ApJ, submitted (arXiv:0912.1077)

Burns, J. O., Roettiger, K., Ledlow, M., \& Klypin, A. 1994, ApJ, 427, L87

Burstein, D. 1979, ApJ, 234, 829

Chung, A., van Gorkom, J. H., Kenney, J. D. P., \& Vollmer, B. 2007, ApJ, 659, L115

Cooper, M. C., Gallazzi, A., Newman, J. A., \& Yan, R. 2010, MNRAS, 402, 1942

Cooper, M. C., et al. 2007, MNRAS, 376, 1445

Cox, T. J., Dutta, S. N., Di Matteo, T., Hernquist, L., Hopkins, P. F., Robertson, B., \& Springel, V. 2006, ApJ, 650, 791

de Vaucouleurs, G. 1948, Ann. d'Astrophys., 11, 247

Dressler, A. 1980, ApJ, 236, 351

Dressler, A. 1984, ARA\&A, 22, 185

Dressler, A. 2004, in Clusters of Galaxies: Probes of Cosmological Structure and Galaxy Evolution, ed. J. S. Mulchaey, A. Dressler, \& A. Oemler (Cambridge: Cambridge Univ. Press), 206

Dressler, A., et al. 1997, ApJ, 490, 577

Fasano, G., Poggianti, B. M., Couch, W. J., Bettoni, D., Kjærgaard, P., \& Moles, M. 2000, ApJ, 542, 673

Font, A. S., et al. 2008, MNRAS, 389, 1619

Gallazzi, A., Charlot, S., Brinchmann, J., White, S. D. M., \& Tremonti, C. A. 2005, MNRAS, 362, 41

Gallazzi, A., et al. 2009, ApJ, 690, 1883

Gilmore, G., Wyse, R. F. G., \& Norris, J. E. 2002, ApJ, 574, L39

Giovanelli, R., \& Haynes, M. P. 1985, ApJ, 292, 404

Gómez, P. L., et al. 2003, ApJ, 584, 210

Graves, G. J., Faber, S. M., \& Schiavon, R. P. 2009a, ApJ, 693, 486

Graves, G. J., Faber, S. M., \& Schiavon, R. P. 2009b, ApJ, 698, 1590

Gunn, J. E., \& Gott, J. R. 1972, ApJ, 176, 1

Hinz, J. L., Rieke, G. H., \& Caldwell, N. 2003, ApJ, 126, 2622

Hogg, D. W., et al. 2003, ApJ, 585, L5

Hogg, D. W., et al. 2004, ApJ, 601, L29

Holden, B. P., et al. 2007, ApJ, 670, 190

Holden, B. P., et al. 2009, ApJ, 693, 617

Kang, X., \& van den Bosch, F. C. 2008, ApJ, 676, L101

Kauffmann, G., et al. 2003, MNRAS, 341, 54

Kauffmann, G., et al. 2004, MNRAS, 353, 713

Kauffmann, G., et al. 2006, MNRAS, 367, 1394

Koopmann, R. A., \& Kenney, J. D. P. 1998, ApJ, 497, L75

Koopmann, R. A., \& Kenney, J. D. P. 2004, ApJ, 613, 851

Kroupa, P. 2001, MNRAS, 322, 231

Larson, R. B., Tinsley, B. M., \& Caldwell, C. N. 1980, ApJ, 237, 692

Laurikainen, E., Salo, H., Buta, R., \& Knapen, J. H. 2009, ApJ, 692, L34

Lewis, I., et al. 2002, MNRAS, 334, 673

Lilly, S. J., Fevre, O. L., Hammer, F., \& Crampton, D. 1996, ApJ, 460, L1

Madau, P., Ferguson, H. C., Dickinson, M. E., Giavalisco, M., Steidel, C. C., \& Fruchter, A. 1996, MNRAS, 283, 1388

Markevitch, M., et al. 2002, ApJ, 567, L27

Martin, N. F., Ibata, R. A., Bellazzini, M., Irwin, M. J., Lewis, G. F., \& Dehnen, W. 2004, MNRAS, 348, 12
McCarthy, I. G., et al. 2008, MNRAS, 383, 593

Moore, B., Katz, N., Lake, G., Dressler, A., \& Oemler, A. 1996, Nature, 379, 613

Moran, S. M., Ellis, R. S., Treu, T., Smith, G. P., Rich, R. M., \& Smail, I 2007, ApJ, 671, 1503

Moster, B. P., Maccio', A. V., Somerville, R. S., Johansson, P. H., \& Naab, T. 2010, MNRAS, 403, 1009

Naab, T., Burkert, A., \& Hernquist, L. 1999, ApJ, 523, L133

Neistein, E., Maoz, D., Rix, H.-W., \& Tonry, J. L. 1999, AJ, 117, 2666

Padilla, N. D., \& Strauss, M. A. 2008, MNRAS, 388, 1321

Park, C., Choi, Y.-Y., Vogeley, M. S., Gott, J. R., \& Blanton, M. R. 2007, ApJ, 658, 898

Pasquali, A., van den Bosch, F. C., Mo, H. J., Yang, X., \& Somerville, R. 2009, MNRAS, 394, 38

Patel, S. G., Kelson, D. D., Holden, B. P., Illingworth, G. D., Franx, M., van der Wel, A., \& Ford, H. 2009, ApJ, 694, 1349

Peng, C. Y., Ho, L. C., Impey, C. D., \& Rix, H.-W. 2002, AJ, 124, 266

Poggianti, B. M., et al. 2006, ApJ, 642, 188

Postman, M. 2005, ApJ, 623, 721

Postman, M., \& Geller, M. J. 1984, ApJ, 281, 95

Quilis, V., Moore, B., \& Bower, R. 2000, Science, 288, 1617

Quinn, P. J., Hernquist, L., \& Fullagar, D. P. 1993, ApJ, 403, 74

Roediger, E., \& Brüggen, M. 2007, MNRAS, 380, 1399

Sandage, A. 1961, The Hubble Atlas of Galaxies (Washington, DC: Carnegie Institution)

Sérsic, J. L. 1968, Atlas de Galaxias Australes (Cordoba, Argentina: Observatorio Astronomico)

Simard, L., et al. 2009, A\&A, 508, 1141

Skibba, R. A. 2009, MNRAS, 392, 1467

Skibba, R. A., \& Sheth, R. K. 2009, MNRAS, 392, 1080

Skibba, R. A., et al. 2009, MNRAS, 399, 966

Smith, S. 1935, ApJ, 82, 192

Smith, G. P., Treu, T., Ellis, R. S., Moran, S. M., \& Dressler, A. 2005, ApJ, 620, 78

Tanaka, M., Goto, T., Okamura, S., Shimasaku, K., \& Brinkmann, J. 2004, AJ, 128,2677

Tasca, L. A. M., et al. 2009, A\&A, 503, 379

Toomre, A., \& Toomre, J. 1972, ApJ, 178, 623

Tran, K.-V. H., et al. 2009, ApJ, 705, 809

Treu, T., et al. 2003, ApJ, 591, 53

Tully, R. B., \& Fisher, J. R. 1977, A\&A, 54, 661

van den Bergh, S. 1976, ApJ, 206, 883

van den Bergh, S. 2009, ApJ, 702, 1502

van den Bosch, F. C., et al. 2008, MNRAS, 387, 79

van der Wel, A. 2008, ApJ, 675, L13

van der Wel, A., Holden, B. P., Zirm, A. W., Franx, M., Rettura, A., Illingworth, G. D., \& Ford, H. C. 2008, ApJ, 688, 48

van der Wel, A., Rix, H.-W., Holden, B. P., Bell, E. F., \& Robaina, A. R. 2009, ApJ, 706, L120

van der Wel, A., et al. 2007, ApJ, 670, 206

Vincent, R. A., \& Ryden, B. S. 2005, ApJ, 623, 137

Vogt, N. P., Haynes, M. P., Giovanelli, R., \& Herter, T. 2004, AJ, 127, 3300

von der Linden, A., Wild, V., Kauffmann, G., White, S. D. M., \& Weinmann, S. 2009, MNRAS, in press (arXiv:0909.3522)

Weinmann, S. M., van den Bosch, F. C., Yang, X., \& Mo, H. J. 2006, MNRAS, 366,2

Weinmann, S. M., et al. 2009, MNRAS, 394, 1213

Whitmore, B. C., Gilmore, D. M., \& Jones, C. 1993, ApJ, 407, 489

Wolf, C., et al. 2009, MNRAS, 393, 1302

Yang, X., Mo, H. J., van den Bosch, F. C., Pasquali, A., Li, C., \& Barden, M. 2007, ApJ, 671, 153

Zwicky, I. F. 1942, ApJ, 95, 555 